\title{
Desensitisation of NO/cGMP signalling in vascular and bronchial smooth muscle
}

\author{
Alexander Lange*, Florian Müllershausen, Andreas Friebe and Doris Koesling
}

\author{
Address: Institut für Pharmakologie und Toxikologie, Bochum, Germany \\ Email: Alexander Lange* - alexander.lange@rub.de \\ * Corresponding author
}

from 2nd International Conference of cGMP Generators, Effectors and Therapeutic Implications

Potsdam, Germany, 10-12 June, 2005

Published: 16 June 2005

BMC Pharmacology 2005, 5(SuppI I):P30 doi:I0.1 186/I47I-2210-5-SI-P30

The messenger molecule NO leads to an enhanced cGMP production by stimulation of NO-sensitive guanylyl cyclase (GC) and thereby causes relaxation of smooth muscle and inhibition of platelet aggregation. In vascular smooth muscle, cGMP synthesis is also stimulated by natriuretic peptides, e.g. ANP, that activate particulate GCcoupled receptors. The amplitude and duration of a cGMP signal critically depends on cGMP degradation by phosphodiesterases with the cGMP-specific cGMP-binding phosphodiesterase type 5 (PDE5) being of major importance in many cells. In platelets, a negative feed-back regulation in NO/cGMP-signalling has been described which is caused by the activation of PDE5. Binding of cGMP to the regulatory GAF-A domain of PDE5 leads to a long-lasting increase in catalytic activity. Consequently, the activation of PDE5 elicits a sustained desensitisation of the signalling pathway in platelets towards further NO stimuli. Recently, a rapid NO-induced desensitisation as a result of PDE5 activation has also been demonstrated in smooth muscle-containing tissues.

Here, we compare the amounts of the principal constituents of the NO/cGMP signalling pathway (GC and PDE5) in two different models of smooth muscle, i.e. aortic and bronchial muscle. The expression levels of NO-sensitive GC and PDE5 and the degree of PDE5 activation were determined. Furthermore, the respective NO-induced cGMP responses were determined in control and NOdesensitized tissues.

Using organ bath experiments we investigate the physiological relevance of the NO-induced desensitisation on various relaxing stimuli. The NO-induced relaxation was impaired by a short term NO pretreatment in aorta and bronchus. In aortic tissue, ANP-mediated vasorelaxation was also impaired showing that desensitisation does not occur on the level of NO-sensitive GC. The direct activation of cGMP-dependent protein kinase caused similar responses of relaxation in NO-pretreated and control vessels. Therefore, the activation of PDE5 is sufficient to explain the observed NO-induced desensitisation of smooth muscle relaxation. 